\title{
Significance of D2-40 and CXCL5 Expression in Urinary Bladder Transitional Cell Carcinoma: An Immunohistochemical Study
}

Rana Abou ElFetouh, Abd ElLatif M. ElBalshy, Nihal S. Zafer, Nashwah M. Omarah. Eman S. Omar

\begin{abstract}
Department of Pathology, Benha faculty of medicine, Benha University, Egypt.

Correspondence to: Rana Abou ElFetouh, Department of Pathology, Benha faculty of medicine, Benha University, Egypt
\end{abstract}

Email:

rana.elsaied@fmed.bu.edu.eg

Received: 1 January 2021

Accepted: 12 June 2021

\begin{abstract}
:
Background: Transitional cell carcinoma remains a challenge in the oncology field, representing an ideal candidate for research on biomarkers that could identify patient's progression and prognosis. D2-40 and CXCL5 have been implicated in progression of many cancers, but their significance in urinary bladder TCC remains unclear. The aim of this study is to assess their possible significance in urinary bladder transitional cell carcinoma. Methods: Immunohistochemistry was performed to examine the expression of D2-40 and CXCL5 in 50 cases of urinary bladder TCC and 10 cases of normal urothelium taken as a normal control. Statistical analysis methods were used to evaluate the relationship between D2-40 and CXCL5 and various clinicopathological parameters. Results: D2-40 was expressed in the lymphatic endothelial cytoplasm of all cases
\end{abstract} high lightening the tumor emboli in cancer lymphatic vessels. CXCL5 was found to be expressed in all urinary bladder TCC cases but not in normal control. The two markers significantly correlated with associated necrosis, tumor grade, T stage, $\mathrm{N}$ stage and presence of H\&E detected LVI. CXCL5 was also positively correlated with presence of associated necrosis and CIS while D2-40 was insignificantly correlated with both. D2-40 detected intralymphatic tumor emboli are associated with lymph node metastasis in a highly significant correlation. Conclusions: The results suggested that CXCL5 might be involved in urinary bladder TCC carcinogenesis. It could be concluded that D2-40 and CXCL5 may have a possible role in urinary bladder TCC progression and prognosis. D2-40 might be considered as a more reliable method in predicting tumor spread and lymph node metastasis.

Keyword: Urinary bladder, transitional cell carcinoma, D2-40, CXCL5, immunohistochemistry 


\section{Introduction}

Bladder cancer has become a common cancer globally, with an estimated 430000 new cases diagnosed in 2012. Worldwide, it is the 7th most common cancer in men and the 17th most common cancer in women. Incidence rates are highest in Europe, the United States and Egypt ${ }^{\mathbf{1}} \mathbf{\&}^{\mathbf{2}}$.

In the Arab world, bladder cancer is frequent where Schistosoma hematobium is endemic, namely: Egypt, Iraq, Sudan, Southern Saudi Arabia and Yemen ${ }^{3}$.

In Egypt, bladder cancer was considered the third most common tumor according to the National Cancer Institute (NCI) registry. However, Transitional cell carcinoma of the urinary bladder represents about $90 \%$ of all bladder cancer cases. Egyptian males have the highest mortality rates $(16.3$ per 100,000$)$ worldwide, which is twice as high as the highest rates in Europe and over 4 times higher than that in the United States ${ }^{\mathbf{4}} \boldsymbol{\&}^{\mathbf{3}}$.

In Egypt, in the Pathology Department of the National Cancer Institute during the time period between the years 2008-2011, urinary bladder cancer is the third most common cancer in both sexes representing
$6.94 \%$ of total malignancies being the second most common cancer in men ${ }^{5}$. Urinary bladder cancer mortality is mainly determined by the initial tumor stage and success of treatment, reflected by progression rates of high-risk non-muscleinvasive $\mathrm{UBC}$ and cure rates of muscleinvasive UBC. In 2008, UBC was the eighth most common cause of cancerspecific mortality in Europe ${ }^{6}$. Nonmuscle-invasive tumors have a high prevalence because their low progression rates allow many patients to survive a long time, while patients with muscle-invasive disease are at significantly higher risk of dying from their disease ${ }^{7}$. Thus, the detection of lymphatic vessel invasion (LVI) at primary diagnosis may predict which patients with T1 UCB are at higher risk for harboring or developing muscleinvasive disease and may be best suited for an earlier and more aggressive form of therapy $(8 \& 9)$.

Podoplanin, a 38-kd mucin-like transmembrane glycoprotein, is highly and specifically expressed in lymphatic endothelial cells, but not blood vessel endothelium. Podoplanin expression has been reported in carcinomas of the skin, 
lung, uterus and esophagus and is highly expressed in oral cancer and some oral premalignancies ${ }^{\mathbf{1 0}}$. D2-40 antibody specifically recognizes human podoplanin and can therefore be used for evaluating its expression in development of neoplasms and lymphatic invasion ${ }^{\mathbf{1 1}}$. However, very few studies have been reported until date demonstrating the significance of D2-40 expression and its potential association with the histopathological characteristics of urinary bladder cancer ${ }^{\mathbf{1 2}}$.

Lack of understanding the mechanisms that govern UBC tumor metastasis and recurrence is a major reason for its high mortality $\quad$ rates $^{\mathbf{1 3}}$. Matrix metalloproteinases (MMPs) play irreplaceable roles in tumor cell extravasations and implantation especially MMP2 and MMP9 that are monitored by various signal transduction pathways targeting tumor metastasis attracting attention to the importance of the tumor microenvironment in disease progression 14 .

Lipopolysaccharide-induced $\quad$ CXC chemokine (CXCL5), also named epithelial-neutrophil activating peptide-78 (ENA-78), is a small protein, belonging to 'ELR+' subgroup of chemokines that binds to its cell-surface receptor CXCR2 to play its role in angiogenesis, tumor migration and invasion by activating the PI3K/AKT pathway-induced up regulation of matrix metalloproteinase ${ }^{15}$. Numerous reports have indicated that CXCL5 plays vital roles in cancer progression, including intrahepatic cholangiocarcinoma, prostate cancer, gastric cancer, and head and neck squamous cell carcinoma ${ }^{16}$. Though studying the Immunohistochemical expression of CXCL5 in UBC to assess its role in tumor cell proliferation, migration and spread is debatable ${ }^{17}$.

Material and Methods:

This is a retrospective study performed on formalin fixed paraffin embedded biopsy specimens of 50 cases of urinary bladder transitional cell carcinoma and 10 cases of normal urinary bladder urothelium taken as control that are obtained during transuretheral dissection of prostate. The specimens are collected from Department of Pathology and Early Cancer Detection Unit (ECDU), faculty of medicine - Benha University from 2011 to 2017.

Histopathological study: Four-microns thick sections were stained by conventional hematoxylin and eosin (H\& E) stain.

Immunohistochemical study: For immunohistochemical (IHC) staining, 10\% 
formalin-fixed, paraffin-embedded, 4micron tissue sections were prepared. They were immunostained for D2-40 concentrated monoclonal antibody (GeneTex, USA) at a dilution of 1:50 and CXCL5, concentrated polyclonal antibody (GeneTex, USA) at a dilution of 1:100. DAP was utilized as a chromogen.IHCstaining was performed, using detection kit (Thermoscientific USA) according to the manufacturers data.

Positive control: subepithelial lymphatics of bladder wall were served as internal positive control and tosillar tissue as external positive control for D2-40 and colonic adenocarcinoma tissue for CXCL5.

Negative control: tosillar tissue for D2-40 and colonic adenocarcinoma for CXCL5 by omitting the primary antibody.

\section{Immunohistochemical assessment:}

Immunohistochemistry with monoclonal antibody D2-40 was performed on intra-tumor and peri-tumoral zone of 50 urinary bladder urothelial carcinoma specimens. A structure with a central lumen, lined by endothelial cells, was taken as positive vessel. D2-40 showed cytoplasmic staining

- Negative: no staining ${ }^{\mathbf{1 8}}$.
The immunohistochemistry results of CXCL5 were evaluated by the immunoreactive score (IRS). The percentage of positive tumor cells (0 $\%=$ negative $, 1-25 \%=1,26-50 \%=2,51-75$ $\%=3$, and $76-100 \%=4)$ and the intensity of immunostaining $\quad$ (none $=0, \quad$ weak $=1$, moderate $=2$, and intense $=3$ ) were assessed, respectively. The IRS of each section was calculated in the percent positive rating multiplied by the intensity rating, ranging from 0 to 12 . IRS score $<6$ is considered as low expression, IRS 6-9 is considered as moderate expression and IRS >9 is considered as high expression.

Statistical analysis: The data were coded, entered and processed on personal computer using statistical program for social science (SPSS) (version 16). The Pearson correlation coefficient was used for statistical analysis. $\mathrm{P}$ value $<0.05$ was considered statistically significant and highly statistically significant when it was $<0.01$.

The procedures followed were in accordance with the ethical standards of the responsible committee on human experimentation and with the Helsinki Declaration of 1975, as revised in 2000. 


\section{Results}

This retrospective study was carried upon 50 selected cases of urinary bladder urothelial carcinoma and 10 cases of normal urothelium taken as control. All cases were examined histologically and immunohistochemically for D2-40 and CXCL5.

Histologically cases were classified into 20 cases (40\%) papillary variant, 24 cases (48\%) conventional transitional carcinoma and 6 cases $(12 \%)$ transitional carcinoma with divergent differentiation (including with squamous differentiation (3), micropapillary (2) and nested (1)). Regarding bilharziasis, 34 cases (68\%) were negative and 16 cases (32\%) were positive. They were graded into: 25 cases (50\%) grade II (Low grade), 15 cases (30\%) grade III (High grade) and 10 cases (20\%) grade IV (High grade). The studied cases were classified according to muscle invasion into 18 cases (36\%) non-muscle invasive and 32 cases (64\%) muscle invasive. According to the $\mathrm{T}$ component of TNM staging system, 7 cases out of the non-muscle invasive cases (38.9\%) are Ta stage and 11 cases $(61.1 \%)$ are $\mathrm{T} 1$ stage. Out of the muscle invasive cases, 16 cases $(50 \%)$ are T2 stage (4 cases (25\%) are T2a and 12 cases $(75 \%)$ are T2b), 9 cases
(28.1\%) are T3 stage (4 cases (44.4\%) are T3a and 5 cases $(55.6 \%)$ are T3b) and 7 cases (21.9\%) are T4 stage (4 cases $(57.1 \%)$ are $\mathrm{T} 4 \mathrm{a}$ and 3 cases $(42.9 \%)$ are T4b). Regarding Lymph node metastasis (LNM), 16 cases (53.3\%) out of the radical cystectomy cases associated with negative lymph nodes (pN0). Fourteen cases $(46.7 \%)$ are positive. Out of the positive cases, 8 cases $(57.1 \%)$ are N1 stage and 6 cases $(42.9 \%)$ are N2 stage. Regarding lympho-vascular invasion (LVI), 21 cases (42\%) were negative and 29 cases (58\%) were positive for tumor emboli. Regarding associated carcinoma in situ (CIS) in nonpapillary urothelial carcinoma cases, 12 cases $(40 \%)$ of non-papillary cases are associated with carcinoma in situ and 18 cases (60\%) not associated with CIS. Regarding necrosis, 42 cases (84\%) show no necrosis and 8 cases (16\%) show necrosis within the tumor area

The results of both antibodies were correlated with different clinicopathological variables of the cases examined and summarized in tables $1 \& 2$.

\section{Immunohistochemical Results:}

Analysis of the relationships between D240 and CXCL5 expression and different clinicopathologic variables was 
summarized and tabulated in tables 1 and 2 . D2-40 detected intralymphatic tumor emboli are associated with lymph node metastasis in a highly significant correlation. Figures (1,2\&3). The two markers significantly correlated with associated necrosis, tumor grade (figure
4\&5), $\mathrm{T}$ stage (figure $6 \& 7$ ), $\mathrm{N}$ stage and presence of H\&E detected LVI. CXCL5 was also positively correlated with presence of associated necrosis and CIS while D2-40 was insignificantly correlated with both.

Table (1): Relation and Correlation between D2-40 expression and clinicopathological parameters

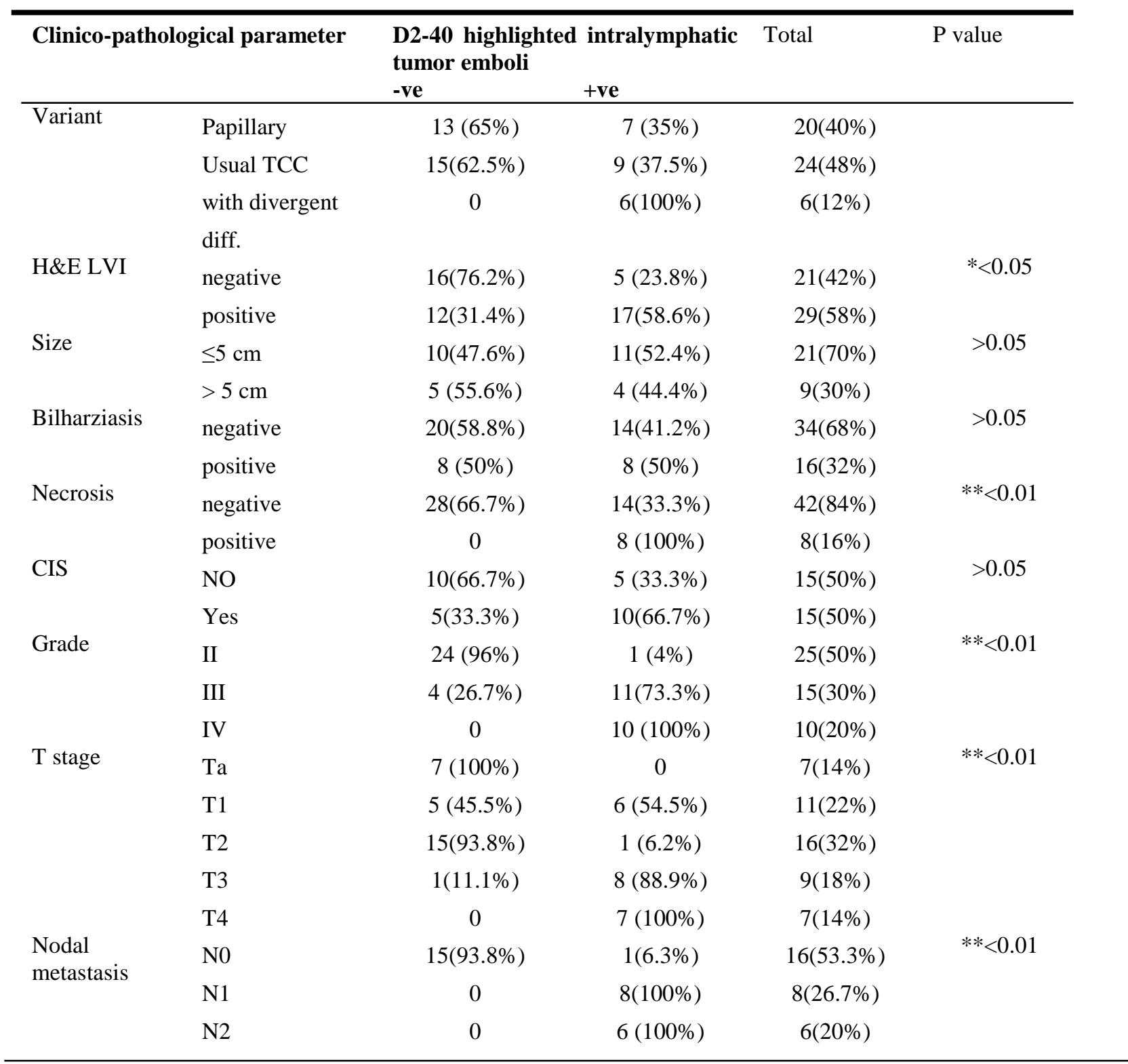


Table (2): Relation and Correlation between CXCL5 expression and clinico-pathological parameters

\begin{tabular}{|c|c|c|c|c|c|c|}
\hline \multicolumn{2}{|c|}{ Clinico pathological parameter } & \multicolumn{3}{|c|}{ CXCL5 expression } & \multirow[t]{2}{*}{ Total } & \multirow[t]{2}{*}{ P value } \\
\hline & & Low & Moderate & High & & \\
\hline \multirow[t]{3}{*}{ Tumor variant } & Papillary & $10(50 \%)$ & $10(50 \%)$ & 0 & 20 & \\
\hline & Usual TCC & 0 & $20(83.3 \%)$ & $4(16.7 \%)$ & 24 & \\
\hline & With divergent diff. & 0 & 0 & $6(100 \%)$ & 6 & \\
\hline \multirow[t]{2}{*}{ Tumor size } & \multirow{2}{*}{$\begin{aligned} & \leq 5 \mathrm{~cm} \\
> & 5 \mathrm{~cm}\end{aligned}$} & - & $15(71.4 \%)$ & $6(28.6 \%)$ & 21 & \multirow[t]{2}{*}{$>0.05$} \\
\hline & & - & $5(55.6 \%)$ & $4(44.4 \%)$ & 9 & \\
\hline \multirow[t]{2}{*}{ Bilharziasis } & Negative & $10(29.4 \%)$ & $19(55.9 \%)$ & $5(14.7 \%)$ & 34 & \multirow[t]{2}{*}{$<0.05^{*}$} \\
\hline & Positive & 0 & $11(68.8 \%)$ & $5(31.2 \%)$ & 16 & \\
\hline \multirow[t]{2}{*}{ Necrosis } & Negative & $10(23.8 \%)$ & $29(69 \%)$ & $3(7.1 \%)$ & 42 & \multirow[t]{2}{*}{$* *<0.01$} \\
\hline & Positive & 0 & $1(11.1 \%)$ & $7(77.8 \%)$ & 9 & \\
\hline \multirow[t]{2}{*}{ CIS } & No & - & $13(86.7 \%)$ & $2(13.3 \%)$ & $15(50 \%)$ & \multirow[t]{2}{*}{$*<0.05$} \\
\hline & Yes & - & $7(46.7 \%)$ & $8(53.3 \%)$ & $15(50 \%)$ & \\
\hline \multirow{3}{*}{$\begin{array}{c}\text { Tumor } \\
\text { Grade }\end{array}$} & II & $10(40 \%)$ & $15(60 \%)$ & 0 & $25(50 \%)$ & \multirow{3}{*}{$\begin{array}{c}*< \\
0.01 *\end{array}$} \\
\hline & III & 0 & $14(93.3 \%)$ & $1(6.7 \%)$ & $15(30 \%)$ & \\
\hline & IV & 0 & $1(10 \%)$ & $9(90 \%)$ & $10(20 \%)$ & \\
\hline \multirow[t]{5}{*}{ T Stage } & Ta & $7(100 \%)$ & 0 & 0 & $7(14 \%)$ & \multirow{5}{*}{$\begin{array}{c}*< \\
0.01 *\end{array}$} \\
\hline & T1 & $3(27.3 \%)$ & $8(72.7 \%)$ & 0 & $11(22 \%)$ & \\
\hline & T2 & 0 & $16(100 \%)$ & 0 & $16(32 \%)$ & \\
\hline & $\mathbf{T 3}$ & 0 & $6(66.7 \%)$ & $3(33.3 \%)$ & $9(18 \%)$ & \\
\hline & T4 & 0 & 0 & $7(100 \%)$ & $7(14 \%)$ & \\
\hline \multirow{3}{*}{$\begin{array}{r}\text { Nodal } \\
\text { metastasis }\end{array}$} & No & $16(100 \%)$ & 0 & $16(53.3 \%)$ & $16(100 \%)$ & \multirow[t]{3}{*}{$* *<0.01$} \\
\hline & N1 & $4(50 \%)$ & $4(50 \%)$ & $8(26.7 \%)$ & $4(50 \%)$ & \\
\hline & N2 & 0 & $6(100 \%)$ & $6(20 \%)$ & 0 & \\
\hline \multirow[t]{2}{*}{ LVI } & Negative & $8(38.1 \%)$ & $12(57.1 \%)$ & $1(4.8 \%)$ & $21(42 \%)$ & \multirow[t]{2}{*}{$* *<0.01$} \\
\hline & Positive & $2(6.9 \%)$ & $18(62.1 \%)$ & $9(31 \%)$ & $29(58 \%)$ & \\
\hline
\end{tabular}




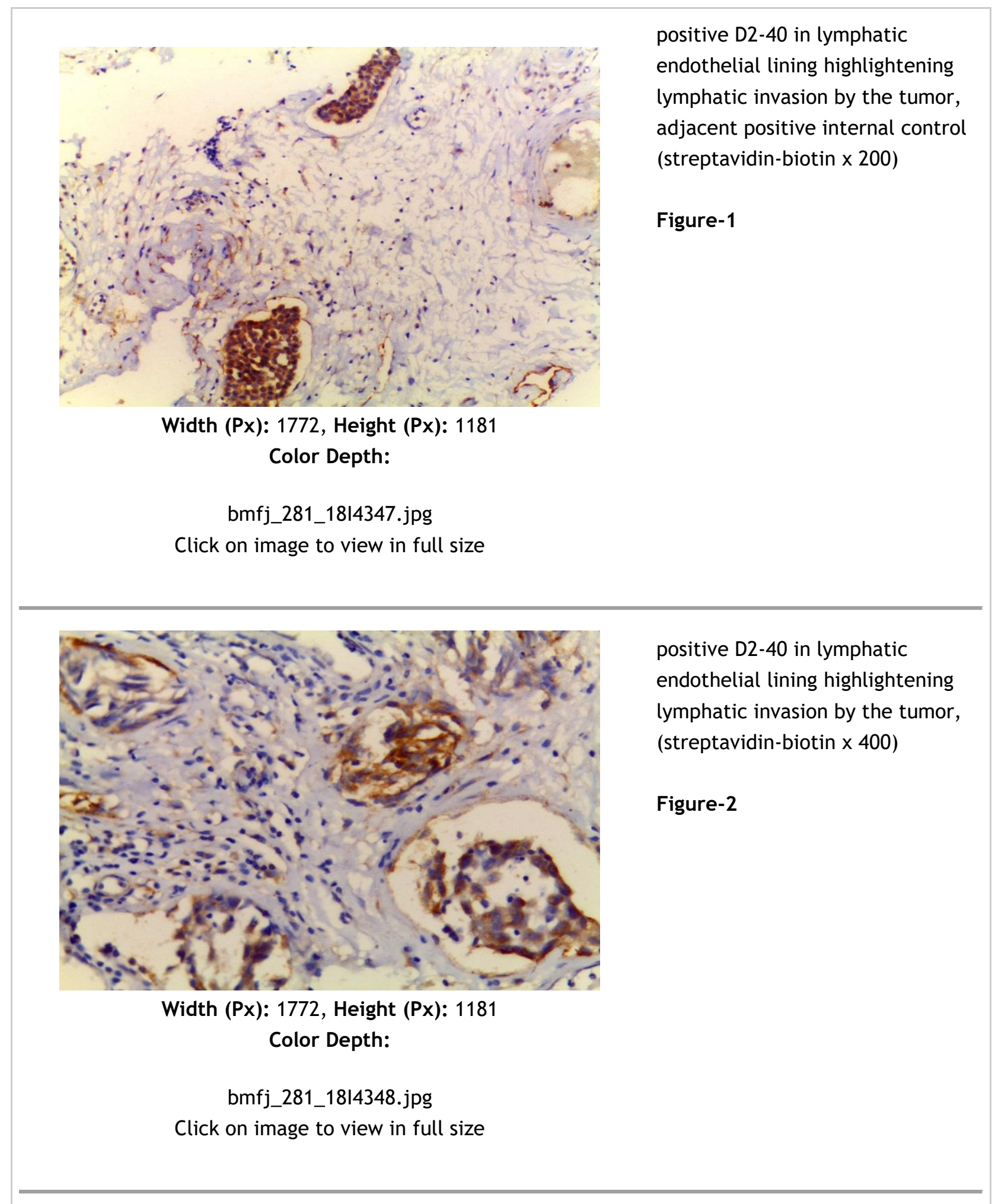




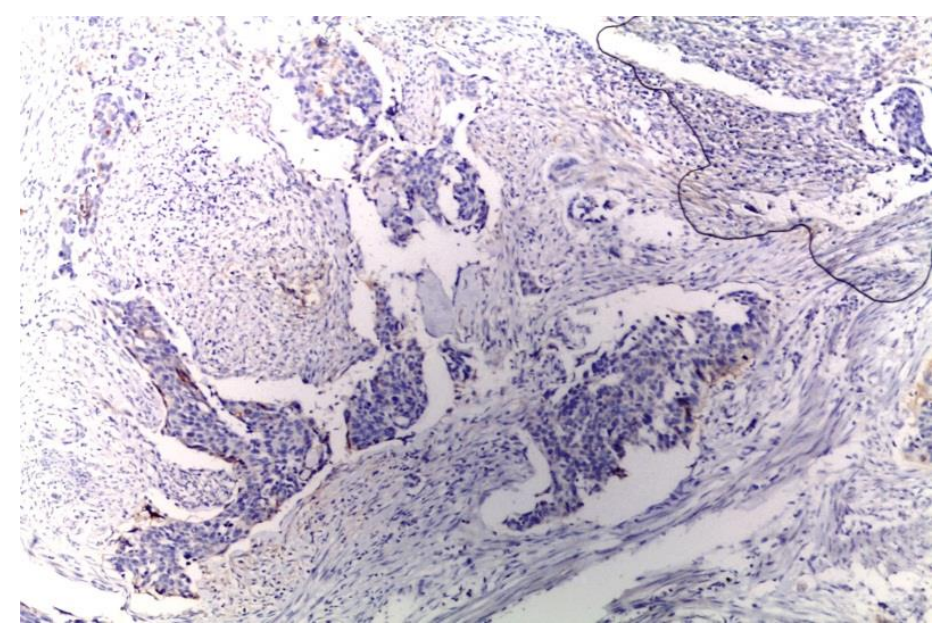

Width (Px): 1772, Height (Px): 1181

\section{Color Depth:}

bmfj_281_18|4349.jpg

Click on image to view in full size negative D2-40 around tumor

clusters (streptavidin-biotin $\times 100$ )

Figure-3

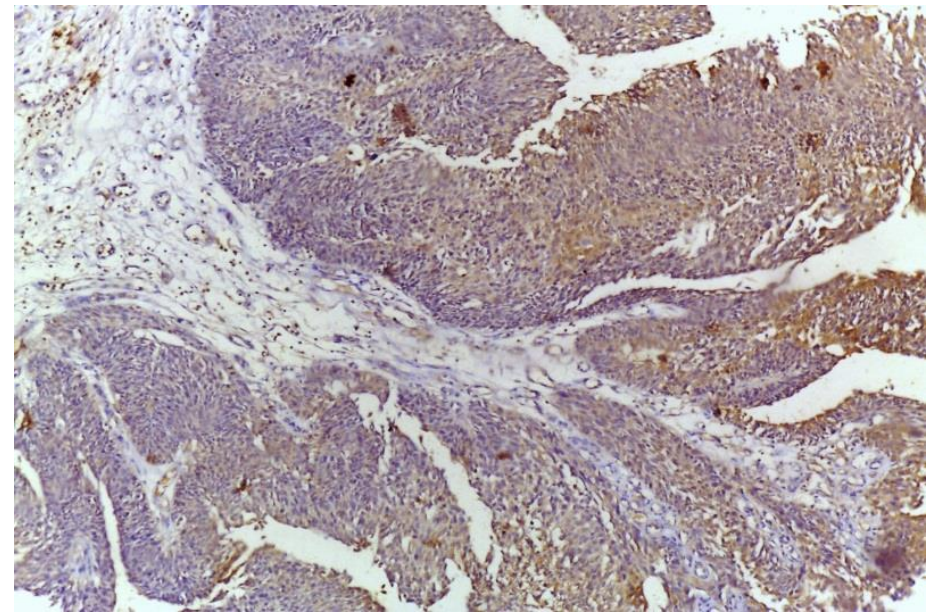

Width (Px): 1772, Height (Px): 1181

Color Depth:

bmfj_281_1814350.jpg

Click on image to view in full size low grade papillary urothelial neoplasm showing low CXCL5 expression (streptavidin-biotin $\mathrm{x}$ 100)

\section{Figure-4}




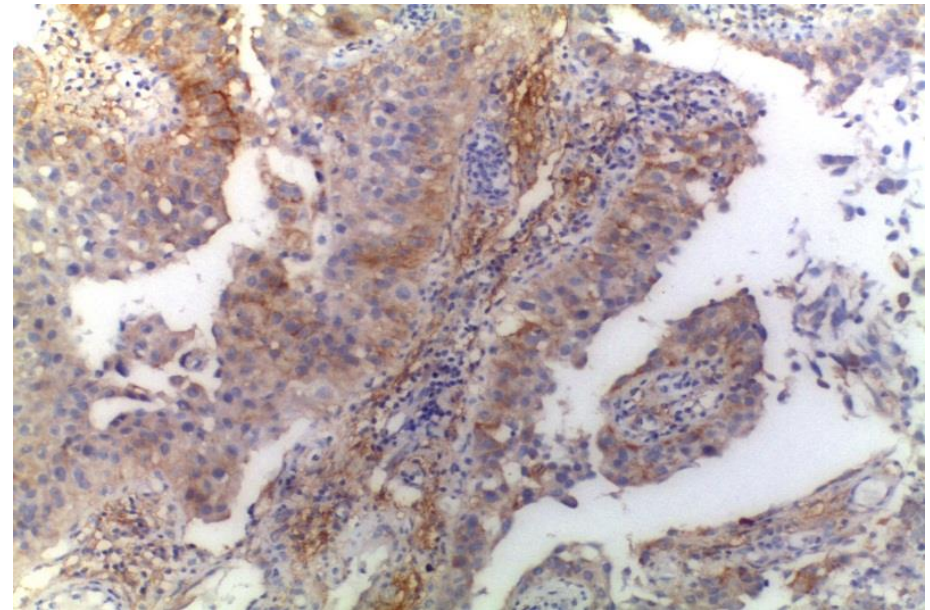

Width (Px): 1772, Height (Px): 1181

Color Depth:

bmfj_281_1814351.jpg

Click on image to view in full size

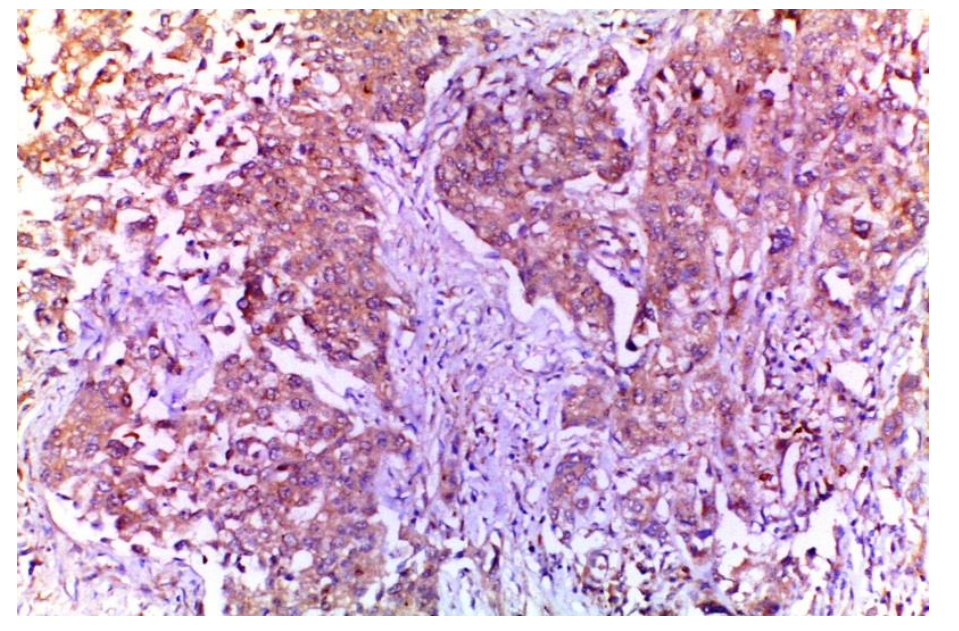

Width (Px): 1772, Height (Px): 1181

Color Depth: grade III papillary urothelial carcinoma showing moderate CXCL5 expression (streptavidinbiotin $x$ 200)

\section{Figure-5}

grade III muscle-invasive urothelial carcinoma showing high CXCL5 expression (streptavidin-biotin $\mathrm{x}$ 200)

\section{Figure-6}

bmfj_281_1814353.jpg

Click on image to view in full size 


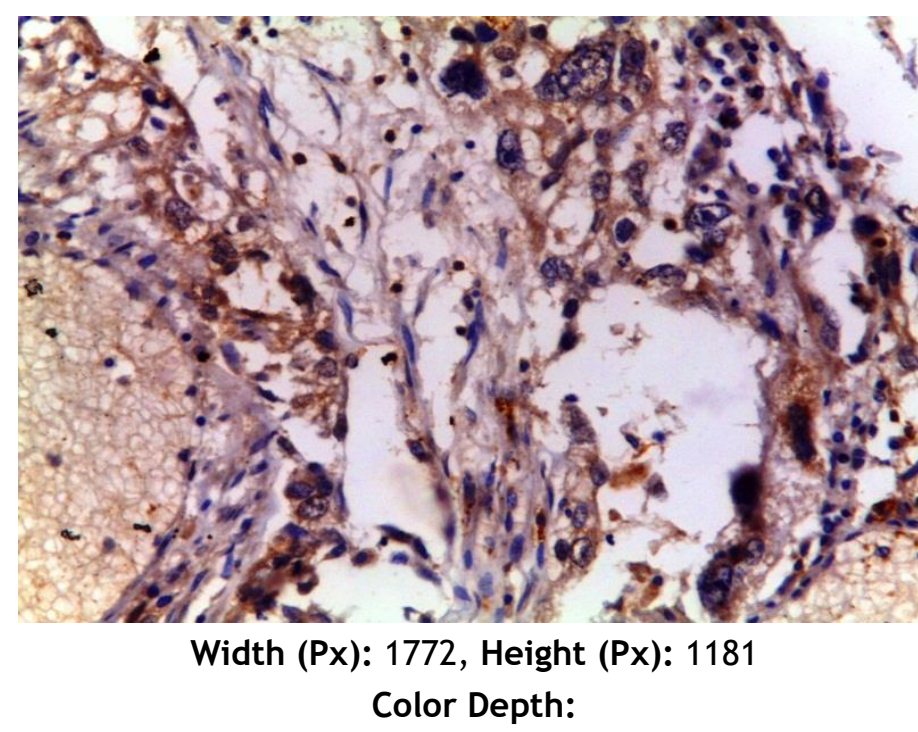

grade IV muscle-invasive urothelial carcinoma showing high CXCL5 expression (streptavidin-biotin $\mathrm{x}$ 400)

\section{Figure-7}

bmfj_281_18|4352.jpg

Click on image to view in full size

\section{Discussion:}

Highly significant statistical positive correlations were found in this study between D2-40 detected intralymphatic tumor emboli and routinely diagnosed LVI by $\mathrm{H} \& \mathrm{E}$ and tumor $\mathrm{N}$ stage which explained by its role in lymphangiogesis and tumor metastasis via its effect on platelet aggregation.

This in line with studies conducted on breast cancer $^{19} \& 20$, a study on rectal neuroendocrine tumors ${ }^{21}$, results concerning primary cutaneous melanomas ${ }^{22} \& 23$, a studyof cutaneous squamous cell carcinoma $^{24}$ and a pancreatic carcinoma study $^{25}$.

Other highly statistically significant positive correlations were found between D2-40 evident lymphatic invasion and tumor $\mathrm{T}$ stage, tumor grade and presence of intratumoral necrosis.

Agreed with that were the results of a study on cutaneous squamous cell carcinoma ${ }^{24}$.

Not matching the studies on breast cancer ${ }^{20}$ and on colorectal cancer ${ }^{26}$ who reached positive significant statistical correlation 
between D2-40 detected lymphatic invasion and tumor $\mathrm{T}$ stage but both found no statistical correlation with tumor grade. The contradictory results of the role of intratumoral and peritumoral lymphatic vessels in tumors differentiation reflects the fact that tumor metastasis and lymphangiogenesis are very complex processes, which can differ signifaicantly in different tumor types or in tumors at different anatomic sites, which still need more studies to clarify it.

One study on endometrial carcinoma ${ }^{27}$ and another study on breast cancer ${ }^{28}$ reached positive significant correlation with tumor grade.

Interestingly, results reached in a study conducted on oral squamous cell carcinoma $^{29}$ suggest an inverse significant correlation between D2-40 highlighted tumor emboli and tumor grade. This seeming contradiction suggesting that invasion in OSCC is not driven by dedifferentiation of squamous cells but by the emergence of a specific tumor cell population with a high self-renewal capacity (cancer stem cells, CSCs), which typically express podoplanin. On the other hand, a study of breast cancer ${ }^{\mathbf{3 0}}$ found no significant correlation with tumor $\mathrm{T}$ stage or grade and another study on oral squamous cell carcinoma ${ }^{31}$ found no significant statistical correlation between D2-40 detected lymphatic invasion and $\mathrm{T}$ stage but reached significant inverse correlation with tumor grade. A statistically significant positive correlation with the $\mathrm{T}$ stage of esophageal adenocarcinoma was reached but no correlation found with the grade ${ }^{32}$. These differences may be attributed to differences in patient selection, methodology or the types of tumors included in the analysis.

This study did not show any significant statistical correlations between D2-40 detected lymphatic invasion and tumor size, associated bilharziasis and presence of CIS.

This is in agreement with the results of a study on cervical squamous cell carcinoma ${ }^{33}$ and a study on colorectal adenocarcinoma ${ }^{34}$.

Unlike the results of a study on rectal neuroendocrine tumors ${ }^{21}$, the results of studies on breast cancer ${ }^{19 \& 20}$ and pancreatic duct carcinoma $^{35}$ reached a statistically significant positive correlation between D240 detected lymphatic invasion and tumor size. This discrepancy may be attributed to the limited number of radically removed specimens in the current study or the differences in "hot spot" areas selections in different studies. 
These study findings also disprove those of a study on oral squamous cell carcinoma ${ }^{29}$ that reached a positive significant correlation between D2-40 expression and tumors associated with leukoplakia. This may be explained by the differences in the tumors natures and their precursors.

Positive immunostaining for CXCL5 was detected in all 50 cases of bladder urothelial carcinoma. However, the normal urothelium in control cases showed lower faint CXCL5 expression compared to transitional cell carcinoma or no expression at all.

The same results stated were reached in bladder cancer studies ${ }^{36 \& 37}$. Also a study on colorectal carcinoma $^{\mathbf{3 8}}$, a study conducted on all kinds of lung cancer tissues compared to normal lung tissue ${ }^{39}$ and a study of cholangiocarcinoma $^{40}$ concluded similar results.

Even though, a study conducted on colorectal specimens disproves this correlation stating that immunohistochemical staining of CXCL5 protein was evident in cancerous as well as non-cancerous tissue with an intensity of staining varying from none to strong ${ }^{41}$. This may be explained by that the non-cancerous cases in the latter study were the resection margins of cancerous cases that show the same underlying molecular changes as colorectal cancer.

No significant statistical correlation was found between CXCL5 expression and tumor size in the present study.

Disagreeing with this finding are the results of a colorectal carcinoma study ${ }^{\mathbf{4 2}}$ and an intrahepatic cholangio-carcinoma study ${ }^{\mathbf{4 3}}$ stating that CXCL5 was significantly correlated with tumor size. This discrepancy may be attributed to limited number of radically removed cases in the current study.

A statistically significant correlation was found between CXCL5 expression \& bilharziasis. This can be explained by the fact that CXCL5 (also named epithelialneutrophil activating peptide-78) is an inflammatory chemokine that binds its receptor $\mathrm{CXCR} 2$ to execute its role in immune response.

This is in line with the study carried on laryngeal squamous cell carcinoma ${ }^{44}$ and the study conducted on colorectal carcinoma specimens ${ }^{45}$. Both reached this significant correlation between CXCL5 expression and presence of associated granulomatous reaction explained by the chemotactic chemokines effect of CXCL5 that neutrophils can potentially produce, either under inflammatory/immune reactions or 
during their activation in more prolonged processes, such as in tumors.

Noteworthy, a statistically highly significant correlation between CXCL5 expression \& presence of necrosis emerged from the current analysis. It is very likely due to the fact that CXCL5 is an angiogenic chemokine that binds to its cell-surface receptor $\mathrm{CXCR} 2$ to execute its roles in tumor progression and growth. Chemokine ligand can deliver anti-apoptotic and proliferative signals, and induce tumournecrosis factor- $\alpha$. The higher the proliferations index, the more the susceptibility of tumor cells to necrosis.

This is consistent with the results of the study on prostate cancer metastasis to bone $^{46}$.

It is crucial to note that a statistically significant correlation was found between CXCL5 expression and presence of associated CIS in the present study. This is could be probably explained by that CIS is a precursor of more aggressive muscle invasive urothelial carcinoma .

In a complete agreement with this result are the results reached in a study of bladder $\mathrm{TCC}^{47}$ and those reached in studies on colorectal carcinoma $^{\mathbf{3 8}}$.
Significantly, a statistical positive correlation was found between CXCL5 expression and urothelial carcinoma grade in this study. This is very likely due to the angiogenic function of CXCL5 mediating the angiogenesis through binding to $\mathrm{CXCR} 2$ and thus affects many cellular functions including tumor growth, proliferation and differentiation.

This fits the results of a bladder cancer study ${ }^{36}$, a study on non-small cell lung cancer $^{48}$ and a study revealing that overexpression of CXCL5 protein had correlation with tumor differentiation in HCC cases ${ }^{49}$.

In contradiction with the present study are the results of the study that found that expression of CXCL5 has a significant reverse correlation with tumor grade in pancreatic cancer ${ }^{\mathbf{5 0}}$ this is interestingly owed to the finding that tumor associated neutrophils (TANs) are found to be involved more in lower tumor grade in which CXCL5, also named epithelialneutrophil activating peptide-78 are more expressed $^{50}$ and those of the study that reached no significant correlation between expression of CXCL5 and tumor grade in metastatic HCC cases ${ }^{\mathbf{4 3}}$. This may be explained by that the nature of tumor in a 
metastatic site may differ than the primary tumor acquiring more genetic alternations.

Statistically significant positive correlation was found between CXCL5 expression and urothelial carcinoma $\mathrm{T}$ stage and $\mathrm{N}$ stage in the present study.

Matching with the current positive correlation between CXCL5 expression and direct and lymphatic metastasis are a study on urinary bladder cancer $^{\mathbf{3 7}}$, a study on intrahepatic cholangiocarcinoma ${ }^{17}$ and studies in breast cancer and non-small cell lung cancer analysis ${ }^{48}$. And in agreement with this finding also are the study results showing that CXCL5 was significantly correlated with Dukes' stage, tumor invasion \& lymph node localization in colorectal cancer $^{42}$ and those reaching a positive correlation between CXCL5 expression and melanoma loco-regional and nodal metastasis $\mathbf{5 1}^{\mathbf{2}}$.

Also there is a study stated that CXCL5 immunoreactivity was occasionally observed in PanIN-1 but the invasive carcinomas showed extensive staining of CXCL5 $^{\mathbf{5 0}}$. These data suggest that CXCL5 is constitutively overexpressed in human pancreatic cancer and that its expression correlates with tumor $\mathrm{T}$ stage.

Unlikely were the results of the study that found expression of CXCL5 protein in all the analyzed tissue from the CRC patients did not correlate with Duke"s stage ${ }^{52}$. This may be explained by discrepancy number of patients and tissue types.

Another highly significant statistical positive correlation was found between CXCL5

expression and LVI.

Agreed with this finding are a study of urinary bladder cancer ${ }^{\mathbf{5 3}}$, a study of melanoma $^{51}$ and a study on intrahepatic cholangiocarcinoma $^{17}$.

\section{Conclusions:}

The results suggested that CXCL5 might be involved in urinary bladder TCC carcinogenesis. It could be concluded that D2-40 and CXCL5 may have a possible role in urinary bladder TCC progression and prognosis. D2-40 might be considered as a more reliable method in predicting tumor spread and lymph node metastasis

\section{References}

1. Malats N, Real FX. Epidemiology of bladder cancer. Hematology/oncology clinics of North America. 2015 Apr 30;29(2):177-89.

2. Torre LA, Siegel RL, Ward EM, Jemal A. Global cancer incidence and mortality rates and trends - an update. Cancer Epidemiology and Prevention Biomarkers. 2016 Jan 1;25(1):16-27.

3. El-Bolkainy MN, Nouh MA, Farahat IG, EI Bolkainy TN, Badawy OM. Pathology of 
cancer. Skin tumors, the National Cancer Institute (NCI), Cairo University. 2013:360-77

4. Lotfy AA, El-Desouky MA, Bahnasy AA, Abdel-fattah HF, Kobaisi MH. Biochemical study of E-Cadherin and SATB1 in Urothelial Bladder Carcinoma of Egyptian Patients. Egyptian Journal of Chemistry. 2020 Dec 28.

5. Ibrahim AS, Khaled HM, Mikhail NN, Baraka H, Kamel H. Cancer incidence in Egypt: results of the national population-based cancer registry program. Journal of cancer epidemiology. 2014 Sep 21;2014.

6. Babjuk M, Burger M, Zigeuner $\mathbf{R}$, Shariat SF, van Rhijn BW, Comperat E, et al ; European Association of Urology (2013). EAU guidelines on non-muscle-invasive urothelial carcinoma of the bladder: update 2013. Eur Urol. 64:639-53. PMID:23827737

7. Witjes JA, Compérat E, Cowan NC, De Santis M, Gakis G, Lebret T, et al; EAU guidelines on muscle-invasive and metastatic bladder cancer: summary of the 2013 guidelines. European urology. 2014 Apr 1;65(4):778-92.

8. Ferlay J, Soerjomataram I, Dikshit R, Eser S, Mathers C, Rebelo M, et al; Cancer incidence and mortality worldwide: sources, methods and major patterns in GLOBOCAN 2012. International journal of cancer. 2015 Mar 1;136(5):E359-86.

9. Cambier S, Sylvester RJ, Collette L, Gontero P, Brausi MA, Van Andel G, et al; nomograms and risk groups for predicting recurrence, progression, and disease-specific and overall survival in non-muscle-invasive stage $\mathrm{Ta}-\mathrm{T} 1$ urothelial bladder cancer patients treated with 13 years of maintenance bacillus Calmette-
Guérin. European urology. 2016 Jan 1;69(1):609.

10. Kawaguchi H, El-Naggar AK, Papadimitrakopoulou V, Ren H, Fan YH, Feng L, et al. Podoplanin: A novel marker for oral cancer risk in patients with oral premalignancy. J Clin Oncol 2008;26:354-60.

11. Kadota K, Huang CL, Liu D, Nakashima N, Yokomise H, Ueno M, et al. The clinical significance of the tumor cell D2-40 immunoreactivity in non-small cell lung cancer. Lung Cancer 2010;70:88-93

12. Gakis G, Todenhöfer T, Braun M, Fend F, Stenzl A, Perner S. Immunohistochemical assessment of lymphatic and blood vessel invasion in $\mathrm{T} 1$ urothelial carcinoma of the bladder. Scandinavian journal of urology. 2015 Sep 3;49(5):382-7.

13. Yan Y, Liang H, Li T, Li M, Li R, Qin X, et al; The MMP-1, MMP-2, and MMP-9 gene polymorphisms and susceptibility to bladder cancer: A meta-analysis. Tumour Biol 35: $3047-$ 3052, 2014

14. Gao Y, Guan Z, Chen J, Xie H, Yang Z, Fan $\mathbf{J}$, et al; CXCL5/CXCR2 axis promotes bladder cancer cell migration and invasion by activating PI3K/AKT-induced upregulation of MMP2/MMP9. International journal of oncology. 2015 Aug 1;47(2):690-700.

15. Lee HJ, Song IC, Yun HJ, Jo DY and Kim S: CXC chemokines and chemokine receptors in gastric cancer: From basic findings towards therapeutic targeting. World J Gastroenterol 20: 1681-1693, 2014

16. Zheng J, Zhu $\mathbf{X}$ and Zhang J: CXCL5 knockdown expression inhibits human bladder cancer T24 cells proliferation and migration. 
Biochem Biophys Res Commun 446: 18-24, 2014.

17. Zhou SL, Dai Z, Zhou ZJ, Chen Q, Wang Z, Xiao YS, et al: CXCL5 contributes to tumor metastasis and recurrence of intrahepatic cholangiocarcinoma by recruiting infiltrative intratumoral neutrophils. Carcinogenesis 35: 597-605, 2014.

18. Martini T, Ströbel $\mathbf{P}$, Steidler A, Petrakopoulou N, Erben P, Bolenz C. Detection of lymphovascular invasion in urothelial carcinoma of the bladder through D240 immunostaining. Der Urologe. Ausg. A. 2015 Jan;54(1):70-5.

19. Abe N, Ohtake T, Saito K, Kumamoto K, Sugino T, Takenoshita S. Clinicopathological significance of lymphangiogenesis detected by immunohistochemistry using D2-40 monoclonal antibody in breast cancer. Fukushima journal of medical science. 2016;62(1):57-63.

20. Abdel-Dayem HM, Ibraheim AT. Expression of D2-40 in Benign and Malignant Breast Lesions and Study the Correlation between Lymphovascular Density and other Prognostic Factors of Breast Cancer. Arch Can Res. 2017;5:1.

21. Kitagawa Y, Ikebe $\mathbf{D}$, Hara T, Kato $\mathbf{K}$, Komatsu T, Kondo F, et al; Enhanced detection of lymphovascular invasion in small rectal neuroendocrine tumors using D2-40 and Elastica van Gieson immunohistochemical analysis. Cancer medicine. 2016 Nov;5(11):3121-7.

22. Feldmeyer L, Tetzlaff M, Fox P, Nagarajan P, Curry J, Ivan D, et al; Prognostic implication of lymphovascular invasion detected by double immunostaining for D2-40 and MITF1 in primary cutaneous melanoma. The American
Journal of Dermatopathology. 2016 Jul 1;38(7):484-91.

23. Creytens D. Prognostic Implication of Lymphovascular Invasion Detected by Double Immunostaining for D-40 and MITF1 in Primary Cutaneous Melanoma: Beware of MITF1 Specificity and Sensitivity in Unusual Melanoma Subtypes. The American Journal of Dermatopathology. 2017 Sep 1;39(9):709.

24. Toll A, Gimeno-Beltrán J, Ferrandiz-Pulido C, Masferrer E, Yébenes M, Jucglà A, et al; D2-40 immunohistochemical overexpression in cutaneous squamous cell carcinomas: a marker of metastatic risk. Journal of the American Academy of Dermatology. 2012 Dec 1;67(6):1310-8.

25. Kurahara H, Shinchi H, Mataki Y, Maemura K, Noma H, Kubo F, et al., Significance of M2polarized tumor-associated macrophage in pancreatic cancer. Journal of Surgical Research. 2011 May 15;167(2):e211-9.

26. Mesina C, Stoean LC, Stoean R, Sandita VA, Gruia CL, Foarfa MC, et al; Immunohistochemical Expression of CD8, CDX2, P53, D2-40 and KI 67 in Colorectal Adenocarcinoma, Conventional and Malignant Colo-rectal Polyps. REVISTA DE CHIMIE. 2018 Feb 1;69(2):419-28.

27. Weber SK, Sauerwald A, Pölcher M, Braun M, Debald M, Serce NB, et al; Detection of lymphovascular invasion by D2-40 (podoplanin) immunoexpression in endometrial cancer. International Journal of Gynecological Cancer. 2012 Oct 1;22(8):1442-8.

28. Pula B, Jethon A, Piotrowska A, Gomulkiewicz A, Owczarek T, Calik J, et al., Podoplanin expression by cancer-associated 
fibroblasts predicts poor outcome in invasive ductal breast carcinoma. Histopathology. 2011 Dec;59(6):1249-60.

29. De Vicente JC, Santamarta TR, Rodrigo JP, García-Pedrero JM, Allonca E, BlancoLorenzo V. Expression of podoplanin in the invasion front of oral squamous cell carcinoma is not prognostic for survival. Virchows Archiv. 2015 May 1;466(5):549-58.

30. Wahal S, Goel M, Mehrotra R. Lymphatic vessel assessment by podoplanin (D2-40) immunohistochemistry in breast cancer. Journal of cancer research and therapeutics. 2015 Oct 1;11(4):798-.

31. Kulkarni, D., Shah, A.A., Ingle, Y., Ramaiah, V.V., Goswamy, K., Naz-Muddebihal, F., et al., 2016. To Study the Regional Difference in Intra-and Peri-tumoral Lymphangiogenesis in Various Histopathological Grades and Stages of Oral Squamous Cell Carcinoma and Correlating with Lymph Node Metastasis Using D2-40: An Immunohistochemical Study. Journal of International Oral Health, 8(4), p.422.

32. Schoppmann SF, Jesch B, Riegler MF, Maroske F, Schwameis K, Jomrich G, et al., Podoplanin expressing cancer associated fibroblasts are associated with unfavourable prognosis in adenocarcinoma of the esophagus. Clinical \& experimental metastasis. 2013 Apr $1 ; 30(4): 441-6$.

33. Xiong Y, Cao LP, Rao HL, Cai MY, Liang LZ, Liu JH. Clinical significance of peritumoral lymphatic vessel density and lymphatic vessel invasion detected by D2-40 immunostaining in FIGO Ib1-IIa squamous cell cervical cancer. Cell and tissue research. 2012 Jun 1;348(3):515-22.

34. Foda AA, El-Hawary AK, Hamed H. Aberrant Expression of Calretinin, D2-40 and Mesothelin in Mucinous and Non-Mucinous Colorectal Carcinomas and Relation to Clinicopathological Features and Prognosis. Pathology \& Oncology Research. 2016 Oct 1;22(4):725-32.

35. Shindo K, Aishima S, Ohuchida K, Fujiwara K, Fujino M, Mizuuchi Y, et al., Podoplanin expression in cancer-associated fibroblasts enhances tumor progression of invasive ductal carcinoma of the pancreas. Molecular cancer. 2013 Dec;12(1):168.

36. Gao Y, Guan Z, Chen J, Xie H, Yang Z, Fan J, et al., CXCL5/CXCR2 axis promotes bladder cancer cell migration and invasion by activating PI3K/AKT-induced upregulation of MMP2/MMP9. International journal of oncology. 2015 Aug 1;47(2):690-700.

37. Zhu X, Qiao Y, Liu W, Wang W, Shen H, Lu Y, et al., CXCL5 is a potential diagnostic and prognostic marker for bladder cancer patients. Tumor Biology. 2016 Apr 1;37(4):4569-77.

38. Yildirim K, Colak E, Aktimur R, Gun S, Taskin MH, Nigdelioglu A, et al., Clinical Value of CXCL5 for Determining of Colorectal Cancer. Asian Pacific journal of cancer prevention: APJCP. 2018 Sep 26;19(9):2481-4.

39. Wu K, Yu S, Liu Q, Bai X, Zheng X, Wu K. The clinical significance of CXCL5 in non-small cell lung cancer. OncoTargets and therapy. 2017;10:5561.

40. Okabe H, Beppu T, Ueda M, Hayashi H, Ishiko T, Masuda T, et al., Identification of CXCL5/ENA-78 as a factor involved in the interaction between cholangiocarcinoma cells and cancer-associated fibroblasts. International journal of cancer. 2012 Nov 15;131(10):223441.

41. Dimberg J, Dienus O, Löfgren S, Hugander A, Wågsäter D. Expression and gene 
polymorphisms of the chemokine CXCL5 in colorectal cancer patients. International journal of oncology. $2007 \mathrm{Jul}$ 1;31(1):97-102.

42. Zhao J, Ou B, Han D, Wang P, Zong Y, Zhu C, et al., Tumor-derived CXCL5 promotes human colorectal cancer metastasis through activation of the ERK/Elk-1/Snail and AKT/GSK3 $\beta / \beta$-catenin pathways. Molecular cancer. 2017 Dec;16(1):70.

43. Zhou SL, Dai Z, Zhou ZJ, Wang XY, Yang GH, Wang Z, et al., Overexpression of CXCL5 mediates neutrophil infiltration and indicates poor prognosis for hepatocellular carcinoma. Hepatology. 2012 Dec;56(6):2242-54.

44. Zhang D, Zhou J, Tang D, Zhou L, Chou L, Chou KY, et al., Neutrophil infiltration mediated by CXCL5 accumulation in the laryngeal squamous cell carcinoma microenvironment: A mechanism by which tumour cells escape immune surveillance. Clinical Immunology. 2017 Feb 1;175:34-40.

45. Tecchio C, Cassatella MA. Neutrophil-derived chemokines on the road to immunity. InSeminars in immunology 2016 Apr 1 (Vol. 28, No. 2, pp. 119-128). Academic Press.

46. Roca H, Jones JD, Purica MC, Weidner S, Koh AJ, Kuo R, et al., Apoptosis-induced CXCL5 accelerates inflammation and growth of prostate tumor metastases in bone. The Journal of clinical investigation. 2018 Jan 2;128(1):24866.

47. Spradling K, Youssef RF. Predictors of Oncologic Outcomes After Treatment of
Oncology Practice 2015 (pp. 577-591). Springer, Cham.

48. Saintigny P, Massarelli E, Lin S, Ahn YH, Chen Y, Goswami S, et al: CXCR2 expression in tumor cells is a poor prognostic factor and promotes invasion and metastasis in lung adenocarcinoma. Cancer Res 73: 571-582, 2013

49. Shen J, Cheng Y, Zhen Q, Mengyi YU. The expressions of chemokine CXCL5 and its receptor $\mathrm{CXCR} 2$ in $\mathrm{HCC}$ and the significance in the prognostic evaluation. The Journal of Practical Medicine. 2015 Jan 1(12):1936-9.

50. Li A, King J, Moro A, Sugi MD, Dawson DW, Kaplan J, et al., Overexpression of CXCL5 is associated with poor survival in patients with pancreatic cancer. The American journal of pathology. 2011 Mar 1;178(3):1340-9.

51. Soler-Cardona A, Forsthuber A, Lipp K, Ebersberger S, Heinz M, Schossleitner K, et al., CXCL5 Facilitates Melanoma CellNeutrophil Interaction and Lymph Node Metastasis. Journal of Investigative Dermatology. 2018 Feb 21.

52. Verbeke H, Struyf S, Laureys G, Van Damme J. The expression and role of CXC chemokines in colorectal cancer. Cytokine \& growth factor reviews. 2011 Oct 1;22(5-6):345-58.

53. Becker C. 5. AuF-Symposium „Urologische Forschung der DGU “in Gießen, 14.-16. November 20135th AuF symposium "Urological research of the DGU" in Gießen, 14-16 November 2013. Der Urologe. 2014 Jan 1;53(1):103-27.

To cite this article: Rana Abou ElFetouh, Abd ElLatif M. ElBalshy, Nihal S. Zafer, Nashwah M. Omarah. Eman S. Omar. Significance of D2-40 and CXCL5 Expression in Urinary Bladder Transitional Cell Carcinoma: An Immunohistochemical Study. BMFJ 2021;38(2): 765-783. DOI: 10.21608/bmfj.2021.56154.1364 